\title{
Therapeutic Induction of Interleukin-10 by Theophylline Administration for the Treatment of Hyposmia
}

\author{
Whitney Hosein, MS ${ }^{1}$, Robert I. Henkin MD, PhD ${ }^{2 *}$ \\ ${ }^{1,2}$ Center for Molecular Nutrition and Sensory Disorders, The Taste and Smell Clinic, 5125 MacArthur Blvd, NW, \#20 Washington, DC 20016, USA
}

*Corresponding author: Robert I. Henkin, MD, Center for Molecular Nutrition and Sensory Disorders, 5125 MacArthur Blvd. NW, \#20 Washington, D.C. 20016, USA.
Received Date: May 22, 2021

Published Date: June 09, 2021

\begin{abstract}
Objective: To determine changes in nasal mucus Interleukin-10 (IL-10) before and after theophylline treatment in hyposmic patients, related to changes in age and diagnoses.

Design: IL-10 was measured in nasal mucus samples of 17 normal subjects and 36 patients with hyposmia of multiple etiologies by use of a sensitive spectrophotometric ELISA assay. Hyposmia is defined clinically by standardized evaluation of impaired gustometry and olfactometry, as well as subjectively self reported by the patient.

Results: Prior to treatment, IL-10 levels in nasal mucus was increased in hyposmic patients compared to controls. Following theophylline administration, IL-10 in hyposmic patients' nasal mucus increased further, correlating with improved subjective taste and smell function. Epithelial IL-10 in the nasal secretions increased linearly with the dosage of theophylline.

Conclusion: Nasal mucus IL-10 increases after theophylline treatment, consistent with IL-10 acting as a taste bud growth co-factor which reduces inflammation locally to maintain the structural integrity of olfactory and taste bud receptors. Measurements of nasal mucus IL-10 provide an important marker for the presence of taste and smell dysfunction and their improvement with theophylline treatment, as well as their return to cytokine homeostasis once the dysfunction is normalized.
\end{abstract}

Keywords: Hyposmia; Hypogeusia; IL-10; Interleukin-10; Theophylline; Inflammation; Nasal mucus; Nasal secretion

\section{Introduction}

Roughly 1 out of every 4 Americans over the age of 40 suffers from an alteration in their olfaction, whereas 1 in 5 of Americans report changes to their gustation after age 40 [1]. The Taste and Smell Clinic of Washington, DC has long investigated and treated this underserved community, which has gained much visibility due to the emergence of the novel coronavirus, SARS-CoV-2. The mechanism of smell and taste loss, hereby referred to as hyposmia and hypogeusia, respectively, is understood as an impairment of the olfactory and gustatory receptors in the nasal/oral mucosa, often as a result of inflammation. This inflammation may be acute, when initiated by a viral infection or head injury; or chronic, as observed in patients suffering from allergic rhinitis, congenital loss or idiopathic etiologies. Inflammation in the nasal and oral mucosa engenders impairment of olfactory and gustatory neurons, resulting in the perceived diminishment of smell and taste, respectively. The source of this inflammation is often due to overproduction of both pro- and anti-inflammatory cytokines due to the aforementioned conditions. Therefore, elimination and suppression of these mediators has been correlated to improved smell and taste function [2].

Interleukin-10 (IL-10) is an anti-inflammatory cytokine present in most tissues of the body with the notable exception of the oral mucosa and salivary glands [2]. Taste cells, however, are capable of expressing IL-10, and are responsible for maintaining structural integrity of the peripheral gustatory system [2]. Cells of the nasopharynx also produce IL-10 in modest amounts [2]. IL-10 is a potent regulator of immunosuppression, and can 
function in a negative feedback loop during viral infection. This cytokine is increased in several disease states such as Multiple Sclerosis, melanoma, metastatic cancers, and during corticosteroid treatment, whereas it is decreased in the airways of patients with severe asthma and chronic pain [3-5]. The Taste and Smell Clinic has been investigating the immunomodulatory relationship of various cytokines in patients with hyposmia and hypogeusia and has found altered cytokine profiles, consistent with chronic inflammation [6]. It has previously been demonstrated that the presence of interleukin-6 (IL-6), a proinflammatory cytokine, was pathologically increased in the saliva of hypogeusic patients, as well as in the nasal mucus of hyposmic patients [6]. Plasma, nasal mucus, saliva and urine levels of IL- 6 were been found to decrease with oral theophylline administration, though it is unclear whether this is correlated to an improvement in olfaction or gustation, due to small sample size [6].

Theophylline was originally prescribed for the treatment of asthma and exercises its effects via multiple pathways, mainly phosphodiesterase (PDE) inhibition, adenosine receptor antagonism, and IL-10 induction via PDE inhibition [7]. Given the anti-inflammatory role of IL-10, its potential regulation in the treatment of hyposmia/hypoguesia via theophylline administration can lend insight into both novel drug targets and its potential dysregulation as the source of these disorders. To better understand this putative relationship, IL-10 is investigated in nasal mucus samples to determine its activity in patients with hyposmia, and whether oral theophylline treatment would alter its concentration therein.

\section{Materials \& Methods}

\section{Normal subjects}

Seventeen volunteers, aged 27-86y, 54 $\pm 5 y$ (mean \pm Standard Error of Mean (SEM)) with normal taste and smell function were studied. These volunteers were either patients who visited The Taste and Smell Clinic in Washington, DC for evaluation of symptoms unrelated to hypogeusia and hyposmia, or voluntary participants from the staff of The Clinic. Subjects were selected in a consecutive manner and included all subjects who volunteered for the study.

\section{Patients}

Thirty six patients, aged $18-84 y, 61 \pm 2 y($ mean \pm SEM) who presented to The Taste and Smell Clinic in Washington, DC for evaluation and treatment of taste and smell loss were included. Patients were all evaluated consecutively at The Clinic from 2008 2018. Patients were: 33 Caucasian, 2 African American, 1 Asian American; 24 men, aged 40-82y, 65 $\pm 3 y$ and 12 women, aged 40-86y, $63 \pm 3 y$. Taste and smell dysfunction was elicited by several clinical events including influenza $(n=14)$, allergic rhinitis $(n=8)$, congenital loss of smell with associated hypogesuia $(n=1)$ head injury $(n=4)$, idiopathic $(n=4)$ chemical exposure $(n=2)$, Bell's Palsy $(n=1)$, and post systemic radiation $(\mathrm{n}=2)$ [8-14]. Patients exhibited taste and smell dysfunction as measured by subjective statements of acuity loss and by clinically standardized gustometry and olfactometry [15]. Subjective statements of acuity loss were quantified by use of a scale from $0-100 \%$, with 100 reflecting total loss of taste or smell function, 0 reflecting no loss and a number between 0-100 reflecting relative degree of loss. Mean \pm SEM of loss degree was measured among all patients and each pathology initiating taste and smell dysfunction.

Gustometry and olfactometry measurements were obtained to evaluate taste dysfunction using an established psychophysical, forced-response staircase technique [15]. Measurements of detection (DT) and recognition (RT) thresholds, magnitude estimation (ME) and hedonics (H) for four tastants [ $\mathrm{NaCl}$ (salt), sucrose (sweet), $\mathrm{HCl}$ (sour) and urea (bitter)] were obtained [15]. Abnormalities of taste function were measured by increased DT or RT above normal (decreased sensitivity) and/or decreased ME (decreased sensitivity) for one or more of the tastants presented [15]. ME was measured on a $1-100 \%$ scale with patients judging taste intensity with respect to tastant intensity experienced prior to onset of taste dysfunction [15]. H varied with respect to perception of pleasantness, unpleasantness or neutrality for each tastant measured on a $1-100 \%$ scale with $+1+100$ indicating pleasantness, $-1--100$ indicating unpleasantness and 0 indicating neutrality [15].

Determination of hyposmia employs measurements of detection (DT) and recognition (RT) thresholds, magnitude estimation (ME) and hedonics $(\mathrm{H})$ for four odorants [Pyridine (dead fish), nitrobenzene (bitter almond), thiophene (petroleum) and amyl acetate (banana oil)] were obtained. In a similar manner, abnormalities of smell function were measured by increased DT or RT above normal (decreased sensitivity) and/or decreased ME (decreased sensitivity) for one or more of the odorants presented [15]. ME was measured on a 1-100\% scale with patients judging intensity with respect to odorant intensity experienced prior to onset of smell dysfunction [15]. H varied with respect to perception of pleasantness, unpleasantness or neutrality for each odorant measured on a $1-100 \%$ scale with $+1+100$ indicating pleasantness, $-1-100$ indicating unpleasantness and 0 indicating neutrality [15].

Theophylline was administered orally, as previously discussed. Oral theophylline was taken by 36 patients in two divided doses for periods of 2-6 months with initial doses starting at 200mg, up to $1000 \mathrm{mg}$ daily. At termination of each treatment period, serum theophylline and taste/smell function were measured. If patients exhibited improvement in taste or smell function they were maintained on that dose; if not, their oral dose was increased by $200 \mathrm{mg}$ and studied again after a period of 2-6 months. During this oral treatment, nasal mucus IL-10, serum theophylline and taste and smell function were measured. If patients exhibited improvement in taste or smell function they were maintained on this dose; if not, their dose was increased by $200 \mathrm{mg}$ and the process was continued until the highest dose of $1000 \mathrm{mg}$ was obtained.

Patients self-collected nasal discharge into a $50 \mathrm{~mL}$ polyurethane tube, refrigerated over a period of up to 3 days prior to their upcoming evaluation at The Clinic, while healthy controls self collected at The Clinic for immediate storage. The samples were stored at $-4^{\circ} \mathrm{C}$ during collection periods and at $-20^{\circ} \mathrm{C}$ upon receipt at The Clinic. Following each treatment period, nasal mucus IL10, 
olfaction and gustation were measured.

Each sample was analyzed by use of a sensitive spectrophotometric ELISA technique obtained from Abcam Inc. (Cambridge, MA). Analysis of duplicate samples agreed within $5 \%$. All analyses were made independent of the knowledge of the status of any subject. Only after all samples were analyzed were results tabulated and samples classified in relationship to subject status. Results were not formulated until all results were analyzed. Results were then calculated with respect to before and after theophylline treatment and analyzed such that mean \pm SEM levels in each category were obtained and results compared using Student $t$ test, with $\mathrm{p}<0.05$ considered significant. Pearson product-moment correlation analyses were also employed with $\mathrm{p}<0.05$ considered significant. ANOVA analyses were also used with $\mathrm{p}<0.05$ considered significant.

No animal models were used in this study or at the clinic. All procedures and studies performed at The Taste and Smell Clinic involving human participants are in accordance with the ethical standards of the institutional review process, and with the 1964 Helsinki Declaration and its later amendments or comparable ethical standards. This study was approved by the institutional review board, Advarra. Informed consent was obtained from all individual participants involved in the study.

\section{Results}

All samples, both patients and controls, contained measurable volumes of IL-10. Mean (SEM) levels in nasal mucus were significantly higher in both treated and untreated patients than in controls $(\mathrm{p}<0.001)$, as shown in (Table 1). Results indicate that patients' nasal mucus IL-10 levels before treatment were significantly higher than in normal subjects by a factor of 11 . After oral theophylline treatment, IL-10 levels in nasal mucus increased significantly $(\mathrm{p}<0.05)$ to levels even greater than in the untreated subjects, to about $15 \mathrm{x}$ higher than normal subjects $(\mathrm{p}<0.001)$. Treated patients were overall shown to increase with treatment to $1.55 \mathrm{pg} / \mathrm{mL}$

TABLE 1: Controls vs. Untreated vs. Orally Treated Hyposmic Patients

\begin{tabular}{|c|c|c|c|}
\hline Sample & Controls (17) & Untreated Patients (36) & Treated Patients (36) \\
\hline Concentration in Nasal Mucus & $.069 \pm 0.01$ & $1.23 \pm 0.18^{\mathrm{a} 1, \mathrm{~d}}$ & $1.83 \pm 0.27^{\mathrm{a} 2, \mathrm{~d}}$ \\
\hline
\end{tabular}

( ) subject number

Treated with respect to (wrt)
a: $p<0.001$
b: $p<0.005$
c: $p<0.01$
$\mathrm{d}: \mathrm{p}<.05$

(Table 2) shows the nasal IL-10 levels in hyposmic patients according to their pathological cause, before and after oral theophylline treatment. Patients diagnosed with PIHH increased significantly above their untreated levels, and congenital patients increased, although not significantly (Table 2). All untreated patients' IL-10 levels were significantly higher than controls, but the patients who decreased significantly with treatment still remain greatly elevated when compared to control values. Patients diagnosed with PIHH increased significantly above their untreated levels, as did patients with hyposmia due to head injury $(\mathrm{p}<0.001)$. All treated patients' IL-10 levels were significantly higher than controls, with the sole congenital patient demonstrating the least increase in treated IL-10 levels.

\begin{tabular}{|c|c|c|}
\hline \multicolumn{3}{|c|}{ TABLE 2: IL-10 by Diagnoses of Untreated vs. Treated Hyposmic Patients } \\
\hline Diagnosis & Untreated & Treated Patients \\
\hline Allergic Rhinitis (8) & $1.00 \pm 0.61^{\mathrm{a} 1}$ & $1.66 \pm 0.15^{\mathrm{a} 2}$ \\
\hline Congenital (1) & $0.21^{\mathrm{a} 1}$ & $0.25^{\mathrm{a} 2}$ \\
\hline Head Injury (4) & $0.458 \pm 0.45^{\mathrm{a} 1}$ & $0.931 \pm 0.27^{\mathrm{a} 2, \mathrm{~d}}$ \\
\hline Idiopathic (4) & $1.48 \pm 0.75^{\mathrm{a} 1}$ & $3.58 \pm 0.60^{\mathrm{a} 2}$ \\
\hline Post Influenza Hyposmia (14) & $1.28 \pm 0.15^{\mathrm{a} 1}$ & $1.81 \pm 0.42^{\mathrm{d}, \mathrm{a} 2}$ \\
\hline Miscellaneous (5) & $2.10 \pm 0.38^{\mathrm{a} 1}$ & $2.26 \pm 1.42^{\mathrm{a} 2}$ \\
\hline
\end{tabular}

( ) subject number

mean \pm SEM $(p g / m L)$

Treated wrt untreated
a: $p<0.001$
b: $p<0.005$
c: $p<0.01$
$d: p<.05$

Untreated wrt Controls

\section{Treated wrt Controls}
a1: $p<0.001$
a2: $p<0.001$
b1: $p<0.005$
b2: $p<0.005$
$c 1: p<0.01$
$c 2: p<0.05$ 
(Table 3) shows changes in hyposmic patients before and after treatment by gender. Levels of IL-10 were significantly higher in men than in women. Levels of IL-10 were not significantly higher in men prior to treatment. With oral theophylline treatment, nasal mucus IL-10 levels increased significantly for men only ( $p<0.001)$. After theophylline treatment in men, IL-10 levels increased significantly compared to both men and women, but both men and wom- en patients had very significantly increased levels when compared to controls $(\mathrm{p}<0.05, \mathrm{p}<0.001$, respectively). Male samples were overall higher when untreated, and with treatment increased significantly, $(p<0.001)$. Women had a modest increase in IL-10 levels compared to men, whose IL-10 levels nearly doubled with oral theophylline treatment.

\begin{tabular}{|c|c|c|}
\hline \multicolumn{3}{|c|}{ TABLE 3: IL-10 in Nasal Mucus of Men \& Women Treated with Theophylline } \\
\hline Gender & Untreated Patients & Treated Patients \\
\hline Women (12) & $0.93 \pm 0.169^{\mathrm{a} 1}$ & $1.01 \pm 0.24^{\mathrm{c}, \mathrm{c}, \mathrm{c}}$ \\
\hline Men (24) & $1.39 \pm 0.29^{\mathrm{a}, \mathrm{a} 1}$ & $2.25 \pm 0.23^{\mathrm{a} 2, \mathrm{c}, \mathrm{d}}$ \\
\hline
\end{tabular}

\section{( ) subject number}

Treated wrt untreated

a: $p<0.001$

b: $p<0.005$

c: $p<0.01$

$d: p<.05$

IL-10 variations in nasal mucus according to age are shown in (Table 4). While IL-10 levels in patients studied by diagnosis and gender increased with theophylline treatment, when studied by

mean \pm SEM $(\mathrm{pg} / \mathrm{mL})$

Untreated wrt Controls

a1: $p<0.001$

b1: $p<0.005$

$c 1: p<0.01$

\begin{tabular}{|c|c|c|}
\hline \multicolumn{3}{|c|}{ TABLE 4: IL-10 by Age in Untreated vs. Treated Hyposmic Patients } \\
\hline Age (years) & Untreated & Treated Patients \\
\hline$>\mathbf{4 0}(\mathbf{2})$ & $2.35 \pm 0.07^{\mathrm{a} 1}$ & $2.49 \pm 2.45^{\mathrm{a} 2}$ \\
\hline $\mathbf{4 0 - 4 9}(\mathbf{7})$ & $1.77 \pm 0.37^{\mathrm{a} 1}$ & $3.15 \pm 1.58^{\mathrm{a} 2}$ \\
\hline $\mathbf{5 0 - 5 9}(\mathbf{3})$ & $0.83 \pm 0.14^{\mathrm{a} 1}$ & $1.65 \pm 1.50^{\mathrm{a} 2}$ \\
\hline $\mathbf{6 0 - 6 9}(\mathbf{1 4 )}$ & $1.75 \pm 0.05^{\mathrm{a} 1}$ & $1.48 \pm 0.42^{\mathrm{a} 2}$ \\
\hline $\mathbf{7 0 - 7 9}(\mathbf{1 1})$ & $1.36 \pm 0.16^{\mathrm{a} 1}$ & $1.14 \pm 1.03^{\mathrm{a} 2}$ \\
\hline$\geq \mathbf{8 0}(\mathbf{4})$ & $0.71 \pm 0.24^{\mathrm{a} 1}$ & $0.81 \pm 0.20^{\mathrm{a} 2}$ \\
\hline
\end{tabular}

( ) subject number

mean \pm SEM $(p g / m L)$

Treated with respect to (wrt)
a: $p<0.001$
b: $p<0.005$
c: $p<0.01$
$d: p<.05$

Treated wrt Controls

a2: $p<0.001$

b2: $p<0.005$

c2: $p<0.05$
M vs. W

a3: $p<0.001$

b3: $p<0.005$

c3: $p<0.05$ age, an increase is seen up until the ages of 60 , after which IL-10 levels appear to decrease.

(Table 5) shows the dose-dependent increase in IL-10 with

Pearson coefficient ( $\mathrm{r}=0.94)$, demonstrating a positive linincreased oral theophylline administration. There is a strong ear relationship between IL-10 levels and theophylline dosage.

\begin{tabular}{|c|c|c|}
\hline \multicolumn{3}{|c|}{ TABLE 5: Increase by Dosage } \\
\hline $\mathbf{n}$ & By Dosage & 1.029 \\
\hline $\mathrm{n}=2$ & $>200$ & 1.069 \\
\hline $\mathrm{n}=8$ & 200 & 1.155 \\
\hline $\mathrm{n}=15$ & $400-600$ & 1.527 \\
\hline $\mathrm{n}=8$ & 600 & 2.315 \\
\hline $\mathrm{n}=3$ & 800 & 0.942133 \\
\hline
\end{tabular}




\section{Discussion}

It has previously been demonstrated that oral theophylline administration inhibits IL-6 expression, and has been observed clinically that treatment with oral theophylline subsequently improved smell and taste dysfunction [16]. Since nasal mucus appears to demonstrate both changes in cytokine expression and subsequent olfactory changes (according to previous investigations at The Clinic), IL-10, also modulated by theophylline, should increase with oral treatment. Theophylline increases IL-10 production in the mast cells of asthmatic airways, and was observed to be increased in mast cells of the nasal passageways [17]. Theophylline presents as an immunomodulatory drug which, even at lower doses, still elicits anti-inflammatory responses without stimulating IL-10 [17]. With respect to immune modulation, women have been shown to have a more sustained, less acute response to infection than men and tend recover from illnesses more slowly, as corroborated by their lower levels of IL-10 without treatment as compared to men, and a minimal increase when treated [18]. Progesterone modulates the production of IL-10, with higher levels of progesterone production corresponding to higher levels of serum IL-10 [19].

Age related smell loss is the greatest contributor, shown by IL-10 in untreated patients decreasing over $30 \%$ after the age of 60 . While the congenital patients were overall younger and had the lowest untreated mean of all diagnoses, $71 \%$ of samples in this study were from patients over 60 years of age, many diagnosed with asthma, diabetes, cancer, or arthritis. "Inflammaging" is a novel term that describes the onset of cytokine dysregulation after the age of 60 , with resultant immune deficiency resulting in hyposmia and hypogeusia [20]. Many patients have reported a transient improvement in smell when treated for immune diseases with prednisone, a potent anti-inflammatory drug and immune suppressor, which also acts to increase circulating IL-10 [21]. This further highlights that hyposmia is mediated by the inflammatory processes of the immune system, such that the initial increase in IL-10 detected in nasal mucus also indicates the presence either localized and systemic inflammation, depending on diagnosis.

The role of inflammation is thus heavily implicated in smell loss, with many patients also demonstrating co-morbidities of inflammatory conditions such as heart disease, arthritis and/or diabetes [22]. IL-10 demonstrates its anti-inflammatory effects by suppression of antigen presenting cells (APCs) during the infective response, thereby inhibiting cytokines, chemokines, and $\mathrm{T}$ cell activation, while activating proliferation, differentiation and endurance of human B cells, as well as production of Immunoglobulin-G, helping to further potentiate the body against infection [23]. Many viruses disrupt the gene that codes for human IL-10 in order to bypass its immune function, so it is important to emphasize that IL-10 is protective at the onset of an infection, but if the pathogen is chronically occurring, then IL-10 will promote pathogenous persistence by way of its anti-inflammatory effects [24]. Certain viruses have evolved to encode for IL-10 analogs, which mimic a systemic increase in circulating IL-10 to the host immune cells, and further induce latency of infection in the host by enhancing human IL-10 anti-inflammatory response at the onset of infection as well as suppression of the pro-inflammatory cytokine storm that would target the source of viral IL-10 [25, 26]. This could account for the overall increased IL-10 presence in nasal mucus after theophylline treatment in certain patients diagnosed with PIHH, especially since antiviral treatment, such as oseltamvir, function via a humoral immune response to increase anti-inflammatory IL-10 expression [27].

\section{Conclusion}

This study demonstrates that IL-10 levels are significantly increased in patients diagnosed with hyposmia, and that with oral theophylline treatment, IL-10 increases further. The initial increase may be correlated with its anti-inflammatory activity in the nasal passages, which in turn can relate to local or systemic inflammatory processes, whether as cause or a result of pathological processes associated with hyposmia. Mechanisms of loss include infection, head injury, drug reaction or allergic conditions, with hyposmia present as a symptom of multiple chronic disease processes involving various organ systems and somatic processes including endocrine, vitamin, trace metal, metabolic, neurological, neurodegenerative, hematological, immunological, and other organ systems and processes.

These results support the biochemical basis of hyposmia such that IL-10 plays a role in pathological processes underlying hyposmia. Oral theophylline increases IL-10 further from control values, as it has been shown to in previous, but unpaired, studies at The Clinic. The pleiotropic role of IL-10 is also highlighted by this study; an increase in endogenous IL-10 is correlated with reduced inflammation, which should result in improved olfaction, however, if human IL-10 gene expression has been corrupted by a persistent or latent virus, then the increases we see are pathological and correlate with the significant increase in both PIHH and head injury [25].

The global emergence of SARS-CoV-2 (COVID-19) has further demonstrated the pleiotropic effects of IL-10, in which the cytokine storm initiated by the novel coronavirus results in pathologically increased IL-10, which acts in a pro-inflammatory manner to stimulate the immune system further and facilitates pathogenic immune escape [28]. Patients recovered from COVID-19 are still shown to have increased IL-10, which may also have a role in the symptoms associated with "long COVID" [29]. Whether their increased IL-10 correlates to associated hyposmic or hypoguesic symptoms, and whether intranasal theophylline will normalize IL10 levels in hyposmic patients, are both topics to be investigated forthwith.

\section{Acknowledgement}

The authors would like to acknowledge Mona Abdelmeguid for assisting in the ELISA preparation and process, as well as in areas of data acquisition. 


\section{Conflict of Interest}

Robert I. Henkin is a member of the board of directors of Cyrano Therapeutics. None of the other authors has a conflict of interest, financial or otherwise with respect to the publication of this manuscript. There is no funding source to be declared.

\section{References}

1. Bainbridge KE, Byrd Clark D, Leopold D (2018) Factors associated with phantom odor perception among US adults: Findings from the National Health and Nutrition Examination Survey. JAMA Otolaryngol Head Neck Surg 144(9): 807-814.

2. Uhlén M (2015) Tissue-based map of the human proteome. Science.

3. Feng, P, Chai J, et al. (2014) Interleukin-10 Is Produced by a Specific Subset of Taste Receptor Cells and Critical for Maintaining Structural Integrity of Mouse Taste Buds. J Neurosci 34(7): 2689-2701.

4. Lu L, et al. (2020) A Potential Role of Interleukin 10 in COVID-19 Pathogenesis. Trends Immunol 42(5): 3-5.

5. Mascali JJ, Cvietusa P, Negri J, Borish L (1996) Anti-inflammatory effects of theophylline: modulation of cytokine production. Ann Allergy Asthma Immunol 77(1): 34-38

6. Henkin RI, Schmidt L, Velicu I (2013) Interleukin 6 in Hyposmia. JAMA Otolaryngol Head Neck Surg 139(7): 728-734.

7. Mitani T, et al. (2018) Theophylline suppresses interleukin-6 expression by inhibiting glucocorticoid receptor signaling in pre-adipocytes. Arch Biochem Biophys 646: 98-106.

8. Henkin RI, Larson AL, Powell RD (1975) Hypogeusia, Dysgeusia, Hyposmia, and Dysosmia following Influenza-Like Infection. Ann Otol Rhinol Laryngol 84(5): 672-682.

9. Church JA, Bauer H, Bellanti JA, Satterly RA, Henkin RI (1978) Hyposmia associated with atopy. Ann Allergy 40(2): 105-109.

10. Henkin RI, Levy LM (2002) Functional MRI of congenital hyposmia: brain activation to odors and imagination of odors and tastes. J Comput Assist Tomogr 26(1): 39-61.

11. Schechter PJ, Henkin RI (1974) Abnormalities of taste and smell after head trauma. J Neurol Neurosurg Psychiatry 37(7): 802-810.

12. Levy LM, Bartsch AJ, Rajan S, Schellinger D, Henkin RI (1995) MRI of olfactory structures: normal subjects and patients with olfactory dysfunction Proceedings of the XV Symposium Neuroradiologicum. Springer, Berlin, Heidelberg 37: 25-26.

13. Henkin RI, Gouliouk V, Fordyce A (2012) Distinguishing patients with glossopyrosis from those with oropyrosis based upon clinical differences and differences in saliva and erythrocyte magnesium. Arch Oral Biol 57: $205-210$.

14. Mossman KL, Henkin RI (1978) Radiation-induced changes in taste acuity in cancer patients. Int J Radiat Oncol Biol Phys 4(7-8): 663-670.
15. Henkin RI, Levy LM, Fordyce A (2013) Taste and smell function in chronic disease: a review of clinical and biochemical evaluations of taste and smell dysfunction in over 5000 patients at The Taste and Smell Clinic in Washington, DC. Am J Otolaryngol 34(5): 477-489.

16. Henkin RI, Hosein S, Stateman WA, Knöppel AB, Abdelmeguid M (2017) Improved Smell Function with Increased Nasal Mucus Sonic Hedgehog in Hyposmic Patients After Treatment with Oral Theophylline. Am J Otolaryngol 38(2): 143-147.

17. Naclerio RM, et al. (1986) Theophylline Reduces Histamine Release During Pollen Induced Allergic Rhinitis. J Allergy Clin Immunol 78(5): 874-876.

18. Vermillion MS, Ursin RL (2018) Production of amphiregulin and recovery from influenza is greater in males than females. Biol Sex Differ 9(1): 24.

19. Kyurkchiev D, Ivanova Todorova E, et al. (2007) Progesterone increases apoptosis and Interleukin-10 secretion by mature monocyte derived dendritic cells. Biotechnology and Biotechnological Equipment 21(4): 468-470.

20. Franceschi C, Garagnani P, Parini P (2018) Inflammaging: a new immune-metabolic viewpoint for age-related diseases. Nat Rev Endocrinol 14(10): 576-590.

21. Mozo L, Suarez A, Gutierrez C (2004) Glucocorticoids Up-regulate Constitutive IL-10 Production by Human Monocytes. Clin Exp Allergy 34(3): 406-412.

22. Zaghloul H, Pallayova M, Al-Nuaimi O, Hovis KR, Taheri S (2018) Association between diabetes mellitus and olfactory dysfunction: current perspectives and future directions. Diabet Med 35(1): 41-52.

23. Perricone C, Shoenfeld N, Agmon Levin N, de Carolis C, Perricone R, et al. (2013) Smell and autoimmunity: a comprehensive review. Clin Rev Allergy Immunol 45(1): 87-96.

24. Miwa T (2019) Clinical practice guidelines for the management of olfactory dysfunction. Auris Nasus Larynx 46(5): 653-662.

25. Rojas JM, Avia M, Martín V, Sevilla N (2007) IL-10: A Multifunctional Cytokine in Viral Infections. J Immunol Res 6(10): 40-54.

26. Gibson AW, Edberg JC, et al. (2013) The Role of IL-10 in Autoimmune Pathology. Austin: Landes Bioscience 1-12.

27. Kakeya H, Seki M, et al. (2014) Efficacy of Combination Therapy with Oseltamivir Phosphate and Azithromycin for Influenza: A Multicenter. Open-Label, Randomized Study. PLoS One 9(3): e91293.

28. Lu L, et al. (2020) A Potential Role of Interleukin 10 in COVID-19 Pathogenesis. Trends Immunol 42(5): 3-5.

29. Humphries IR, Trez Cd, et al. (2007) Cytomegalovirus exploits IL-10-Mediated Immune Regulation in the salivary glands. J Exp Med 204(5): 1217-1225. 